\title{
La Lingüistica de Corpus y la Enseñanza del Inglés (como lengua extranjera): ¿Un matrimonio forzado?
}

\author{
Mauricio Véliz Campos*
}

\section{Resumen:}

El presente artículo aborda, a partir de la revisión crítica de la literatura pertinente, la relación entre la Lingüística del Corpus (LC) y la enseñanza del inglés, la que para muchos aparece como virtualmente automática, mientras que para otros aparece como una herramienta puramente complementaria. El objetivo del artículo es presentar el valor específico de los supuestos contenidos en dos posiciones que parecen estar en conflicto respecto de la eventual transferencia de los hallazgos hechos a partir de la investigación de LC a la sala de clases de inglés como lengua extranjera.

El artículo procura, además, explorar las implicancias de la (tensionada) relación entre la LC y sus transferencias pedagógicas en el contexto de de la(s) forma(s) de enseñanza de inglés en instituciones formadoras de profesores de inglés y del sistema escolar nacional.

Palabras clave: Lingüística del Corpus, corpus/corpora

\section{Abstract:}

This article addresses, based upon a critical survey of the relevant literature, the relationship between Corpus Linguistics (CL) and EFL teaching. Such a relationship comes across as an automatic one, whilst for others it constitutes a mere complementary tool.

The article aims to describe the intrinsic value of the assumptions contained in two -apparently conflicting- positions towards the possible transfer of findings that $\mathrm{CL}$ has produced to the EFL classroom. Likewise, It is intended to explore the implications of such a tense relationship on the training of future teachers of English and the national school context.

Key words: Corpus Linguistics, corpus/corpora

* Académico Universidad Católica Silva Henríquez. Departamento de Humanidades. mveliz@ucsh.cl 


\section{Introducción}

Los estudios lingüísticos han abordado las descripciones -y también prescripciones- a partir de diferentes enfoques, desde aquellos cuyos intereses se centraban en los orígenes y la evolución de las lenguas hasta aquellos que, principalmente gracias al advenimiento de la creciente capacidad de almacenamiento de los computadores, pretenden dar cuenta del comportamiento concreto de las lenguas por medio de la observación y estudio de vastas colecciones de habla/texto (cuasi)espontánea(o).

Los estudios basados en corpus son, contrario al imaginario prevaleciente, bastante más antiguos de lo que se cree; por cierto, es la aparición de las recientes herramientas tecnológico-computacionales la responsable de tanto la cientificidad estadística de dichos estudios, como también de su creciente masificación.

Sin que explícitamente se empleara el término Lingüística del Corpus, ya a fines del siglo XIX, Kading (1897), reunió un corpus del alemán de más de 11 millones de palabras con el fin de determinar, entre otras cosas, secuencias de letras en mencionada lengua. Asimismo, algunas de las figuras más prominentes del periodo estructuralista entre las cuales se cuenta, por ejemplo, Boas (1940), también hicieron uso de corpora con el objeto de observar el comportamiento de las lenguas amerindias.

En la década de los treinta se llevó a cabo un número importante de trabajos en donde se analizó corpora con el fin de establecer frecuencias léxicas en uso de habla real (Palmer, 1933; Palmer \& Hornby, 1937). Dicho trabajo se caracterizó por ser conducido e interpretado por profesionales cercanamente conectados con la enseñanza del inglés.

En 1960, Quirk diseñó y llevó a cabo un estudio denominado Survey of English Usage, trabajo que más tarde Starvic digitalizó y complementó con el famoso corpus de Brown, dando origen a lo que Leech (1991) con- 
sidera como un recurso casi sin comparación para aquellos interesados en estudiar la oralidad del inglés.

El uso de diarios (un tipo de corpus), cuidadosamente elaborados, por parte de aquellos lingüistas dedicados a los procesos de adquisición de lenguaje han sido, hasta ahora, una fuente riquísima de material lingüístico a estudiar. Los estudios longitudinales en esta línea investigativa, de una tradición también bastante extensa, han hecho uso de importantes colecciones de enunciados contenidos en diarios, por ejemplo Brown (1973) y Bloom (1970)

Chomsky, el lingüista que en su mérito personal marcó un hito en la lingüística moderna, postula críticas implícitas a la lingüística del corpus, pues para él, el núcleo investigativo del lingüista radica en lo que él denomina competencia, es decir, el conocimiento que posee el hablante de (las reglas de) su lengua; por otro lado, lo que él denominó performance, corresponde a una pobre manifestación de la riqueza contenida en la competencia.

En la siguiente sección se presentan algunas consideraciones preliminares sobre el uso de corpora en estudios lingüísticos, junto con las ventajas que presenta la LC. Más tarde, se pretende abordar más directamente el debate sobre la (eventual) transferencia de los estudios de la LC a la enseñanza del inglés. Finalmente, se proporciona una postura que recoge algunos de los aspectos más sobresalientes del debate en cuestión, junto con ejemplos concretos aplicables al contexto de la sala de clases de inglés.

\section{Consideraciones preliminares}

Los corpora de hoy en día se ven compelidos a responder a tres requerimientos básicos generales: (i) tamaño, aunque éste depende de la motivación de su construcción y sus usos concretos; (ii) equilibrio interno, pues debe responder al periodo que dice representar, a la variedad dialectal escogida y con una estructura interna en una proporción tal de tipo de texto que permita validar conclusiones emergentes a partir de él, y (iii) facilidad de uso, dependiendo una vez más del uso que tendrá. Además de lo anterior, se suma a estos requerimientos la dificultad que existe en los derechos de autor, especialmente cuando son varios los participantes de un acto comunicativo oral escogido para el corpus y el cuidado en la codificación de los datos lingüísticos.

El impacto de la LC ha permitido que virtualmente todos los sub-componentes lingüísticos se vean afectados por los desarrollos tecnológicos, 
entre ellos se cuentan la fonética, tanto articulatoria, como acústica y auditiva; lo mismo aplica a la fonología, de acuerdo a la cual se indaga sobre la distribución de tanto segmentos como suprasegmentos en una lengua determinada. La gramática constituye probablemente una de las áreas que ha permitido una prolífica producción de literatura especializada; sin embargo, el estudio del lexicon es el que ha dado origen a una serie de diccionarios y estudios acotados que, en ocasiones, expresan pretensiones de convertirse en materiales innovadores para propósitos pedagógicos.

Así como lo establece Leech (1991), para muchos el uso de corpus en los estudios pre-chomskyanos eran concebidos como única fuente de evidencia de la teoría lingüística, desde los más entusiastas exponentes como Harris (1951) hasta los más moderados como Hocket (1948). Chomsky a este respecto presenta una serie de reparos y valora, en su defecto, la introspección, es decir, el espacio en donde yace la competencia lingüística; sólo ella nos permite desambiguar enunciados, además de establecer qué oraciones son gramaticalmente correctas o incorrectas.

Respecto de las ventajas que normalmente se esgrimen a favor de los estudios basados en corpus, se puede mencionar las siguientes:

(i) los datos emergentes del corpus son observables y objetivos, (Leech, 1992);

(ii) la gran mayoría de las oraciones contenidas en corpus son gramaticalmente correctas, (Labov, 1969), en clara oposición a las declaraciones de Chomsky (1968);

(iii) la capacidad de procesamiento de datos lingüísticos es cada vez mayor, en velocidades crecientemente más altas y márgenes de error virtualmente bordeando el cero;

(iv) los ejemplos de datos lingüísticos corresponden a habla real -a pesar de ser este término lo suficientemente contencioso para muchos estudiosos- encontrados en (pequeños) contexto(s);

(v) la facilidad de acceso a corpora que puede ser analizado y trabajado es tal que ya no se requiere de herramientas tecnológicas demasiado sofisticadas; el acceso puede ser ahora personal y doméstico.

Tal ha sido la efervescencia con la cual se ha asumido esta etapa renovada y tecnologizada de la LC que, de acuerdo a Johansson (1991), el número de estudios importantes basados en corpus ha pasado de 10 hasta 1965 a más de 320 que se realizaron sólo en un lapso de 15 años, de 1985 a 1991. Ello ha ido de la mano de la construcción de corpora

(254


en inglés, de los cuales el primero es el ya mencionado Survey of English Usage, en 1960; luego viene el denominado Brown University Corpus of American English, primer corpus computarizado en la década de los sesenta; más tarde en la década de los setenta se produjo el LancasterOslo/Bergen Corpus of British English; en la década de los ochenta se creó Collins-Birmingham University International Language Database (COBUILD), trabajo a partir del cual surge el diccionario que lleva su nombre. Ya en la década de los noventa aparece un proyecto denominado Bank of English, el British National Corpus (BNC) y el International Corpus of English, entre otros.

\section{LC y su transferencia pedagógica}

El debate aquí tratado se basa principalmente en los escritos académicos contenidos en Controversies in Applied Linguistics, de Barbara Seidlhofer (2003), en donde se presenta dos posturas en relativa oposición respecto de la eventual transferencia pedagógica del constructo subyacente a la LC a la sala de clases de inglés como lengua extranjera.

A este respecto, se presenta a Carter \& McCarthy, junto con Gavioli \& Aston como defensores de la relación entre LC y enseñanza del inglés, mientras que Prodromou \& Cook cuestionan esta relación de descripción lingüística y prescripción pedagógica.

Una de las características que suele presentarse como ventaja de las descripciones lingüísticas, con sus subsecuentes implicancias pedagógicas, es que el habla recogida es natural, 'real' -aunque como lo establece el mismo Carter (1998), el término real está extraordinariamente cargado de connotaciones positivas. Lo anterior, en clara contraposición a las formas de habla contenidas en textos.

Lo que parece ser aun más venturoso es consignar que la forma de habla británica informal, así como lo hacen McCarthy \& Carter (1995), es inglés 'real'. Es cierto: las concordancias ampliamente usadas por los aficionados al estudio de corpora dan cuenta de ciertas verdades lingüísticas que, en muchas ocasiones, van en contra de enseñanzas tradicionales. Para ello el ejemplo (1), tomado de Corpus of Spoken Professional American English, da cuenta del uso de any en formas tradicionalmente menos atendidas en las salas de clases de inglés como lengua extranjera y en textos de gramática de naturaleza más introspectiva. 
La lingüistica del corpus y la enseñanza del Inglés (como lengua extranjera): ¿un matrimonio forzado? / Mauricio Véliz Campos

(1)

This is going to be a test like any other test, like, for example working with you..If there are any questions about how we're going to

and I didn't receive any materials for the November meeting

and it probably won't make any difference. I mean, that's the next

You can do it any way you want.

Do you want to ask any questions? make any comments? I don't have any problem with that. I'm just saying if they make any changes, they would be minor changes. I think we ought to use any kind of calculator. I think that way

I see it and it doesn't make any sense to me, but I can take that

What conclusions can you draw about the use of any?

What are the three main uses of any in order of frequency?

De esta muestra se desprenden importantes conclusiones relacionadas con (i) el uso de any y (ii) la frecuencia de cada uno de ellos. La segunda conclusión es la que probablemente demuestre de manera elocuente cuán alejadas están las impresiones de muchos textos y profesores. Sin embargo, dichas muestras 'reales' están supeditadas a la pertenencia de un individuo a la comunidad cultural de la lengua en cuestión. En otras palabras, no se puede hablar como un hablante de inglés británico de registro informal si no se pertenece a dicha comunidad de práctica, particularmente si añadimos a dicha exigencia los aspectos segmentales y prosódicos del inglés, especialmente si al analizar una muestra significativa de los planes de estudio de los casi 100 programas de formación de profesores de inglés en Chile, se observa una gradual desaparición de la cátedra de fonética y fonología inglesas. Es por ello que merece la pena preguntarse si suena más 'raro' (McCarthy \& Carter, 1995:207) utilizar un léxico bookish o hablar con un léxico propio de la variedad y registro mencionados, pero con un ritmo, acentuación y entonación claramente foráneos.

Por otro lado, tal como lo cuestiona Prodromou (1996), ¿hasta qué punto puede, bajo la premisa anteriormente señalada, enseñar inglés 'real' el profesor no nativo? En el mismo sentido, el principio subyacente a la enseñanza de inglés 'real', a partir de muestras pertenecientes a una variedad dialectal determinada y a un registro particular, subsume, quizás de manera indirecta, el supuesto de que nuestros estudiantes de inglés como lengua extranjera aprenden dicha lengua para comunicarse con 
nativos de la lengua inglesa. Subsume, a su vez, que el 'hablante nativo' -concepto cada más elusivo en la reflexión antropo-lingüística- se constituye invariablemente en nuestro modelo, empoderándolo a expensas del profesor no nativo. Dicha presunción resulta extraordinariamente falaz cuando uno observa que las crecientes cifras de hablantes de inglés como segunda lengua y como lengua extranjera superan largamente los 400 millones, por lo que el adoptar una variedad dialectal que represente el inglés 'real' debe ser repensado a la luz del valor ya virtualmente intrínseco del inglés de ser la lingua franca de los tiempos actuales.

Es probablemente cierto que los profesores de lenguas nos fascinamos con los procesos de habla natural, y como ello -parcialmente, por supuesto- se encuentra contenido en corpora, tendemos a pensar que es ello lo que debe penetrar las aulas y los textos de lengua. En este sentido el ejemplo (2), tomado del corpus de Nottingham, debiera hacernos reflexionar sobre la aplicabilidad y pertinencia pedagógica de dichos materiales:

(2)

D' you want a biscuit

Erm

Biscuit

Er yeah

(pause)

All right

Yeah

(pause)

Didn't know you used boiling water

Pardon

Didn't know you used boiling water

Don't have to but it's....they reckon it's erm

(pause)

Tony was saying they should have the heating on by about Wednesday.

Frente a ejemplos como (2), parece mucho más sensato pensar en material adaptado, considerando el grupo etáreo y el nivel de los estudiantes, además de sus necesidades, tanto individuales como grupales.

Pese al ejemplo anterior, los enunciados y diálogos situacionales contenidos en corpora parecen de mucha utilidad al lingüista analista del discurso -más que para el estudiante de inglés como lengua extranjera, pues en ellos se presentan una serie de elementos tales como aproximativos, por ejemplo, there were about twenty or so people at the dinner (tomado de CANCODE, Cambridge and Nottingham Corpus of 
Discourse in English), marcadores textuales, elipsis, interrupción en los participantes del acto comunicativo, silencios, retroalimentación, entre otros. El ejemplo (3), tomado de CANCODE, contrasta dramáticamente con el ejemplo (4), el que se presenta como habla construida para un propósito pedagógico particular:

Ejemplo (3): En una peluquería

A: Do you want to come over here?

B: Right, thanks (3 secs) thank you

A: Tea or coffee?

B: Can I have a tea, please?

A: Do you want any sugar?

$B: E r$, no milk or sugar, just black thanks

C: Right.

B: I hate it when your hair just gets so, you know a bit long

C: yeah and it's just straggly.

C: Right

B: It just gets to that in - between stage

C: Yeah doesn't it where you think oh I just can't stand it any more (2 secs)

I think when it's shorter it tends to, you notice it growing more anyway

C: Mm you know it tends to grow all of a sudden

Ejemplo (4): En la peluquería

Jane $\quad$ :...Oh, yes, my husband's wonderful!

Sally : Really? Is he?

Jane : :Yes, he's big, strong, and handsome!

Sally :Well, my husband isn't very big, or very strong... but he's very intelligent.

Jane : Intelligent.

Sally : :Yes, he can speak six languages.

Jane : Can he? Which languages can he speak?

Sally : He can speak French, Spanish, Italian, German, Arabic and Japanese.

Jane : Oh!....My husband's very athletic.

Sally : :Athletic?

Jane :Yes, he can swim, ski, play football, cricket and rugby...

Sally : Can he cook?

Jane :Pardon?

Sally :Can your husband cook? My husband can't play sports....but he's an excellent cook. 
Jane : Is he?

Sally :Yes, and he can sew, and iron...he's a very good

husband.

Jane : Really? Is he English?

(Hartley and Viney, Streamline English Departures, Unit 14, 1978)

Carter (1998) ofrece razones adicionales para el empleo de corpora para propósitos pedagógicos, entre las que se encuentra el hacerse un estudiante consciente de la lengua aprendida, para lo cual la exploración de la lengua en sus diferentes registros, variedades y contextos puede resultar ser tremendamente útil. Carter ofrece, además, un camino intermedio para trabajar con textos de habla contenidos en corpora, a los que él denomina text modification and remodelling, el que consiste, como lo sugiere su nombre, en hacer modificaciones menores al texto con el fin de hacerlo más amigable y enseñable, por ej.

(5)

A: And I came over Mistham by the reservoirs, nice it was.

B: Oh, by Mistham, over the top, nice run.

A: Colours are pleasant, aren't they?

B: Yeah.

A: Nice run, that.

\section{(CANCODE)}

(6)

A: And I came over the village of Mistham by the reservoirs, it was nice it was.

B: Oh, you came over the top by Mistham. That's a nice journey.

A: The Colours are pleasant, aren't they?

B: Yes.

A: It was a nice journey, that.

Texto modificado

Esta postura adoptada por Carter es denominada 'moderada' o incluso pusilánime por Cook (1998), quien sí reconoce, por ejemplo, que uno de los grandes aportes de la LC es mostrar que la lengua en uso no se circunscribe al dominio de reglas gramaticales, en combinación armónica con ítemes léxicos; es, más bien, una vasta colección de colocaciones, principio al que suscriben Willis (2003), Larsen-Freeman (2003), McCarthy \& Carter (1995). En este sentido se ha masificado, tanto a nivel académico como de órganos responsables de políticas públicas, la virtual convicción de que los grados de comprensión de un texto en inglés están exclusi- 
va y matemáticamente relacionados con el número y tipo de palabras -según frecuencia- que el estudiante sepa. No obstante, dicha relación, puramente matemática, ignora aspectos fundamentales inherentes al ejercicio pedagógico, a saber tratamiento de las expectativas de los estudiantes por parte del profesor, las diferencias individuales, dentro de las cuales se insertan las estrategias de aprendizaje; actitudes del profesor y estudiantes, la diversidad cultural, entre otros. Además, así como lo señala Cook (1998), los despliegues de concordancias de un corpus en términos de frecuencias léxicas o rango muestran habla producida, pero ignora otro aspecto igualmente importante como es la percepción de habla y su interpretación, aspectos ahora atendidos por la pragmática. Dichos despliegues de concordancias, muy útiles por cierto, no dan cuenta de otros aspectos tan verdaderos del uso de la lengua como son la eventual infrecuencia de un ítem en particular, pero su potencial utilidad o pertinencia pedagógica; o la frecuencia de un ítem y su estrechez de rangos contextuales. Por ejemplo, expresiones como go Dutch, que aparece sólo dos veces en BNC; and l am a Dutchman, que aparece sólo una vez; o Dutch courage, Dutch cap que no aparecen ni una vez, constituyen un grupo de expresiones que por su naturaleza semántica, además de la relación con el contexto de nuestros estudiantes en Chile, debiera ser incluido en nuestras clases; ello, pese a su frecuencia, Gavioli \& Aston (2001)

Por último, si asumimos que las muestras de habla contenidas en corpora se deben convertir en modelos para nuestros estudiantes, especialmente sobre la lógica de registros matemáticos de frecuencia, ¿Cuál es el espacio de actividades curriculares que apuntan a la composición creativa? ¿Cuál es el rol de la literatura en los programas de formación de profesores de inglés en Chile, aparte de aprendizajes esperados meramente actitudinales?

\section{Conclusiones}

A la luz de los antecedentes entregados respecto del debate aquí tratado, se puede concluir lo siguiente:

(i) los estudios y análisis basados en corpus llegaron para quedarse (McCarthy \& Carter, 1995). Ello no sólo se basa en el número de estudios de esta naturaleza, ver 2.0, sino también en la riqueza del debate que la LC ha suscitado en la comunidad académica y de actores relevantes. En este incremento de producción académica, las herramientas tecnológicas de almacenamiento y procesamiento de datos lingüísticos han jugado, indubitablemente, un rol fundamental. 
(ii) existe ya una diversificación bastante amplia de tipos de corpora, en donde la indagación sobre el lexicon supera los otros usos disponibles.

(iii) las aportaciones que la LC es capaz de hacer son extraordinariamente ricas en términos de que dan cuenta objetiva respecto, no sólo de aspectos puramente matemáticos tales como frecuencia de ítemes lingüísticos, sino también de la naturaleza léxica y formulaica de la lengua inglesa, en particular.

(iv) parte importante de los hallazgos desafían, a partir de la evidencia empírica entregada por los análisis de concordancias, de manera elocuente un número significativo de creencias (lingüísticas) provenientes de textos tradicionales escritos sobre la base de la introspección.

(v) parece extraordinariamente peligroso aseverar que el habla real corresponde a una variedad dialectal, en un registro determinado, McCarthy \& Carter (1995), desacreditando otras variedades, otros registros y una verdad ya incontrovertible: nuestros estudiantes de pedagogía en inglés, en conjunto con sus estudiantes en el sistema escolar usarán, probabilísticamente hablando, el inglés con otros nonativos. Por ello, surge la inevitable pregunta ¿por qué esmerarse en modelar nuestra enseñanza sobre la base de una variedad que muy probablemente no escucharán en contextos reales?

(vi) aquello que se concibe como habla real, por el hecho de provenir de un corpus, está invariablemente recubierto de un contexto sociocultural virtualmente intransferible a la sala de clases. Si a ello se le suma aspectos propiamente lingüísticos como son elementos prosódicos de habla que, dada las características de nuestros estudiantes y estudios afines, no son adquiridos en los programas de formación regulares, debiéramos cuestionar la pertinencia de un énfasis en 'habla real', entendida como uso léxico real, pero encapsulado en un envoltorio suprasegmental foráneo. La premisa de habla real basada en el hablante nativo exclusivamente hace a ese tipo de habla in-enseñable por parte del profesor no nativo, junto con desempoderarlo socialmente.

(vii) pareciera ser que los valiosísimos datos que entrega la LC se prestan, con mayor facilidad, al análisis del discurso o análisis conversacional, más que al uso inmediato de este tipo de material en la sala de clases. Ello, pues dicho material suele estar lleno de elipsis, interrupciones en la toma de turnos, marcadores textuales, partidas falsas, vacilaciones, etc. Es por esto que resulta sensato pensar en posturas intermedias 
La lingüistica del corpus y la enseñanza del Inglés (como lengua extranjera): ¿un matrimonio forzado? / Mauricio Véliz Campos

como la que ofrece Carter en su sugerencia de modificación de texto y remodelaje, ver ejemplos (5) y (6)

(viii) los datos entregados por concordancias de corpus en términos de frecuencias léxicas no debieran ser el único criterio que determine qué se enseña y qué no.

\section{Referencias}

Bloom L. (1970)

Boas F. (1940).

Brown R. (1973).

Carter, R. (1998).

Cook, G. (1998).
Language development: form and function in emerging grammars, Cambridge, MA: MIT Press.

Race, language and culture. New York: Macmillan.

A first language: the early stages. Cambridge. MA: Harvard University Press.

'Orders of reality: CANCODE, communication, and culture', ELT J., 52: 43-56.

'The uses of reality: a reply to Ronald Carter', ELT J., 52: 57-63.

Gavioli, L. \& Aston, G. (2001). 'Enriching reality: language corpora in language pedagogy', ELT J., 55: 238-246.

Harris Z. (1951).

Methods in structural linguistics. Chicago: University of Chicago Press.

Hockett C. (1948).

Johansson S. (1991).

Kading J. (1879).

Labov V. (1969).

'A note on structure'. International Journal of American Linguistics, 14: 269-71.

'Times change and so do corpora'. En Aijmer \& Altenburg (eds.) English corpus linguistics: studies in honour of Jan Svartvik. London: Longman.

Häufigkeitswörterbuch der deutschen Sprache. Steglitz: Publicado de manera privada.

'The logic of non-standard English'. Georgetown Monographs on Language and Linguistics, 22: 1-22, 26-31.

Larsen-Freeman, D. (2003). Teaching language: from grammar to grammaring. Heinle \& Heinle Publishers.

Leech G. (1991).
'The state of the art in corpus linguistics'. En Aijmer K. \& Altenberg B. (eds.). English corpus linguistics: studies in honour of Jan Svartvik. London: Longman. 
Leech G. (1992). $\quad$ 'Corpora and theories of linguistic performance'. En Svartvik J. (eds.) Directions in corpus linguistics. Berlin: Mouton de Gruyter.

McCarthy, M. \& Carter, R. (1995).

'Spoken grammar: what is it and how can we teach it? ELT J.; 49: 207-218.

Palmer, H. (1933). Second interim report on English collocations.

Tokyo: Institute for research in English teaching.

Palmer, H. \& Hornby, A. (1937).

Thousand- Word English. London: George Harrap.

Prodromou, L. (1996). 'Correspondence', ELT J.; 50: 88-89

Quirk R. (1960). 'Towards a description of English usage'. Transactions of the Philological Society: 40-61.

Seidlhofer, B. (2003). Controversies in applied linguistics. Oxford: Oxford University Press.

Willis, D. (2003). Rules, patterns and words. Cambridge: Cambridge University Press. 